7.

7.
Die internationale Bedeutung der deutschen Reichstagswahl
Von Heinz Neumann (Berlin)

Wenn Parlamentswahlen für uns Kommunisten niemals bloße Stimmkämpfe sind, sondern Gradmesser für die Kräfte im Klassenkampf, so gilt dies mehr als jemals zuvor für die gegenwärtigen Reichstagswahlen in Deutschland. Die Wahlschlacht, die durch das Auflösungsdekret der halbfaschistischen Regierung Brüning in Deutschland entfesselt wurde, ist im Grunde ein Aufmarsch aller kämpfenden Klassenkräfte, eine Formierung der sozialen Fronten, die für die gesamte künftige Entwicklung Europas von größter Bedeutung ist . . .

Der Youngplan erweist sich als ein unlösbarer kapitalistischer Widerspruch, der zu einer gewaltsamen Entladung drängt. Das erste Opfer dieses Widerspruchs ist die bürgerliche Demokratie, die wimmernd am Boden liegt. Die Bourgeoisie, von der äußersten Rechten bis zu ihrer linkesten Koalitionspartei, den gestrigen Demokraten, erkennt, daß die Weimarer Republik, die parlamentarische Demokratie, vollkommen außerstande ist, die Durchführung des Youngplans zu sichern und den drohenden Ansturm der Revolution niederzuhalten. Darum beseitigt die deutsche Bourgeoisie die letzten Überreste des demokratischen Systems. Darum setzt sie die Weimarer Verfassung durch den Diktaturparagraphen 48 außer Kraft. Darum läßt sie durch den Reichspräsidenten Hindenburg das Parlament auflösen. Darum entfesselt sie mit ihrer ganzen politischen und organisatorischen Macht die faschistischen Tendenzen im Lande und bewaffnet die nationalsozialistischen Terrorbanden gegen die Arbeiterklasse.

Der Fußtritt, mit dem das deutsche Finanzkapital die Koalitionsregierung Hermann Müller stürzte, war das erste Signal zur Errichtung der faschistischen Diktatur. Die Sozialdemokratie in den Ministersesseln soll in eine Sozialdemokratie ohne Ministersessel verwandelt werden, um desto rücksichtsloser gegen das revolutionäre Proletariat vorzugehen. Die Eigenart der Faschisierung Deutschlands besteht darin, daß die entscheidenden Gruppen des Finanzkapitals, mit Hindenburg an der Spitze, selbst die Diktatur aufrichten und sich gleichzeitig der Sozialdemokratie und der faschistischen Wehrverbände dabei bedienen. Die Fragestellung lautet für die deutsche Bourgeoisie nicht: Faschismus oder Sozialdemokratie? - sondern: Faschismus mit Sozialdemokratie. So entsteht die eigenartige Situation, daß in der Reichsregierung die bürgerlichen Hindenburg-Parteien, in der Preußenregierung die gleichen Hindenburg-Parteien mit der Sozialdemokratie und in der Thüringer Regierung die gleichen HindenburgParteien mit den Nationalsozialisten sitzen. Alle Parteien in Deutschland, mit Ausnahme der Kommunisten, sind also Koalitionsparteien, Regierungsparteien. Alle Parteien in Deutschland, mit Ausnahme der Kommunisten, sind also Youngparteien, die, gestützt auf die Regierungsmacht, den Youngplan auf Kosten der werktätigen Massen erfüllen.

Daran ändert nichts die betrügerische Behauptung der Nationalsozialisten, sie seien Feinde des Youngplans. In Thüringen, wo bereits das „Dritte Reich“ Hitlers am 
Leben ist, wird der Youngplan genauso durchgeführt wie in Preußen, wo die Sozialdemokratie regiert. Die nationalen Phrasen der Faschisten sind ebenso heuchlerisch wie die sozialen Phrasen der Sozialdemokratie. Beide Hilfstruppen der Bourgeoisie verkaufen das werktätige Volk Deutschlands sowohl an das deutsche als auch an das ausländische Kapital. Beide plündern und knebeln das deutsche Proletariat in gleicher Weise....

Die deutschen Reichstagswahlen sind eine Massenentscheidung für oder gegen den Faschismus. Durch ganz Europa geht eine Welle der faschistischen Reaktion, die als letztes Zufluchtsmittel der herrschenden Klassen gegen die Radikalisierung des Proletariats, gegen den Ansturm der sozialistischen Revolution geschleudert wird. Während in Italien, Spanien, Litauen und Polen die bestehenden faschistischen Diktatoren durch den Druck der Weltwirtschaftskrise und den Klassenkampf des Proletariats erschüttert werden, sind in Jugoslawien, Österreich und Finnland neue faschistische Diktaturen errichtet worden. In Deutschland, dem größten Industriestaat Europas, tobt der Kampf zwischen Faschismus und proletarischer Revolution. Die bankrotte bürgerliche Demokratie und ihre Hauptvertreterin, die Sozialdemokratische Partei, steht in diesem Kampf bedingungslos an der Seite des Faschismus. Die Arbeitermassen, die sich von der Sozialdemokratie abwenden, stehen in diesem Kampf mit wachsender Entschiedenheit auf der Seite der proletarischen Revolution. Die Reichstagswahlen sind ein Kraftmesser für die unvermeidlich herannahenden Entscheidungskämpfe zwischen Revolution und Faschismus in Deutschland, die von ausschlaggebender Bedeutung für die zukünftige Entwicklung in ganz Europa sein werden. . . .

[Internationale Pressekorrespondenz Nr. 69 vom 15. August 1930, S. 1677]

8.

Die Reichstagswahlen und die Kommunistische Partei

Von W. Gollmick (Berlin)

Die Nationalsozialisten aber glauben, daß ihre Stunde heranreift. Sie spüren sehr deutlich, daß sich die parlamentarische Demokratie in Todeskrämpfen windet, daß in den Massen das Parlament seine Autorität verloren [hat], daß die Massen nach anderen Auswegen suchen. So schlagen sie überall los mit schamloser Demagogie. Das „Dritte Reich“ Hitlers soll Heil und Erlösung allen Notleidenden bringen. „Freiheit und Brot" ist ihre zweite Parole zum frechen Massenbetrug. „Zerbrecht die Ketten des Young-Planes“ - „Kämpft gegen Reaktion und Marxismus“ - „Heraus aus den alten Parteikadavern“ - „Nieder mit dem raffenden Börsenkapital“ - das ist eine kleine Blütenlese aus ihren politischen Schlagworten, mit denen sie versuchen, in die Betriebe einzudringen, mit denen sie hoffen, bei dem 3-Millionen-Heer der Erwerbslosen, unter den proletarisierten Mittelschichten, bei den von der Agrarkrise hin und her geschüttelten Kleinbauern Stimmen und Positionen zu gewinnen.

Welches Gewicht die Großindustrie und der Großgrundbesitz sowie die Bankkapitäne dem Hakenkreuzgesindel beilegen, davon zeugt eine Tatsache: Allein für Versammlungszwecke hat die Nationalsozialistische Arbeiterpartei rund 1 Million Mark zur Verfügung. Aber großspurig kündet Hitler an, daß Wahlflugblätter und Aufrufe der Nazis in einer Auflage von 10 bis 15 Millionen erscheinen werden und 
daß die Flugblätter und Schriften der Nazis „buchstäblich in jedes deutsche Haus“ gratis und franko geliefert werden.

Es kommt noch hinzu, da $\beta$ die Nazis in skrupelloser Manier alle wirksamen Agitations- und Propagandamethoden der KPD für ihre eigenen Zwecke nachäffen und in ihren Wahlanweisungen direkt zu stehen haben, daß Betriebs- und Häuserblockzeitungen „nach dem Muster der KPD“ überall anzufertigen sind. Ja, sie schlagen sogar vor, die Zeitungen zu "tarnen" und einfach die Namen der kommunistischen Betriebs- und Häuserblockzeitungen zu nehmen. So ist ganz klar, daß die Kommunistische Partei in ganz anderer Weise als bisher diesem zweiten Hauptfeind der deutschen Arbeiterklasse zu Leibe gehen muß .....

[Internationale Pressekorrespondenz Nr. 70 vom 19. August 1930, S. 1705]

9.

12.9. 1930

Was ist die nationalsozialistische Partei?

Von Werner Hirsch (Berlin)

Ihrer sozialen Struktur nach stützt sich die Hitler-Partei vor allem auf kleinbürgerliche Schichten, hat aber auch einen nennenswerten Anhang unter der Arbeiterschaft, vor allem unter den bisherigen Arbeiterwählern der alten bürgerlichen Parteien. Ihren ersten großen Aufschwung nahm die nationalsozialistische Bewegung in der Inflationszeit 1923. Dann setzte in dex Stabilisierungsperiode ein rapider Rückgang ein, während heute seit Jahresfrist eine starke erneute Offensive dieser Haupttruppe des Faschismus in Deutschland sich vollzieht. Die Nationalsozialistische Partei wird zu einem Sammelbecken derjenigen Massen, die sich von der offen bürgerlichen Führung der anderen Parteien loslösen, ohne schon zum revolutionären Proletariat, zum Kommunismus zu stoßen. Der Haupthebel, der diese Aufwärtsbewegung der Hitler-Partei auslöst, ist selbstverständlich die Politik des Sozialfaschismus. Die tiefe Enttäuschung über die verräterische Politik der Sozialdemokratie in den Massen, auch über den Rahmen der eigentlichen sozialdemokratischen Anhänger hinaus, schafft die Voraussetzung für die Hitler-Partei, mit ihrer schrankenlosen sozialen und nationalen Demagogie Anhänger in starkem Ausmaß zu werben. Das Wachstum des Nationalsozialismus hat somit eine doppelte Bedeutung: Es widerspiegelt die Krise des kapitalistischen Systems, insofern sich im Lager des extremen Faschismus die Streitkräfte der Bourgeoisie zusammenballen und damit die Klassengegensätze klaffender und drohender werden als zuvor. Es widerspiegelt diese Krise aber zugleich dadurch, daß die Massen, die die Hitler-Partei vorübergehend an sich zu ziehen vermag, nur deshalb dem Nationalsozialismus verfallen, weil er sich ihnen im Gewande einer angeblich antikapitalistischen, angeblich revolutionären, angeblich sozialistischen Partei naht.

Diese skrupellose Demagogie der Nationalsozialisten steht im schärfsten Widerspruch zu dem wirklichen Inhalt ihrer Politik. Wie steht die Nationalsozialistische Partei zum Kapitalismus? In ihrer äußeren Agitation bezeichnet sie sich als Arbeiterpartei, die gegen den Kapitalismus kämpfe. Aber Hitler selbst hat folgende Formulierung programmatisch geprägt:

„Es gibt überhaupt kein kapitalistisches System. Die Unternehmer haben sich auf Grund ihrer Tüchtigkeit an die Spitze gearbeitet, und auf Grund dieser Auslese, die 
wiederum nur die höhere Rasse bedeutet, haben sie ein Recht, zu führen. Jeder Wirtschaftsführer wird sich verbitten, einen Betriebsrat mitreden zu lassen. “. .

Die praktische Funktion der Nationalsozialisten in der deutschen Innenpolitik ist nach alledem nichts anderes als die einer Schutzgarde des Unternehmertums, die bisweilen noch schamloser und offener zum Streikbruch gegenüber den Wirtschaftskämpfen des Proletariats aufruft als selbst die reformistische Bürokratie.

Nicht anders steht es mit der angeblich nationalen Politik der Hitler-Partei. In der Programmschrift ihres Reichstagsabgeordneten Feder, die vom Jahre 1919 an in verschiedenen Auflagen erschien, steht ein Satz, der sich gegen die Unterdrückung derjenigen Deutschen richtet, die durch die Friedensverträge von Versailles und Saint Germain unter die Botmäßigkeit anderer Staaten gekommen sind. Es heißt dort:

"Wir verzichten auf keinen Deutschen in Sudetendeutschland, in Südtirol, in Polen, in der Völkerbundskolonie Österreich. "

In den neuen Auflagen dieser Schrift seit 1929 sind die Worte „in Südtirol“ plötzlich gestrichen. Um diese Zeit hatte sich das Einschwenken der Nationalsozialistischen Partei in eine völlig englisch-italienisch orientierte Außenpolitik vollzogen. So konnte ein nationalsozialistischer Parteisekretär am 19. April 1929 wörtlich erklären :

„Gegen die Bundesgenossenschaft Italiens wendet man ein, daß Mussolini Südtirol bedrücke. Das ist aber nur eine von den Freimaurern erfundene Lüge. Außerdem kommt es auf das Schicksal von einigen armseligen Südtirolern nicht an.“...

Diese ganze Politik mit dem Ziel brutalster Konterrevolution, schrankenloser faschistischer Diktatur im Dienste des deutschen und internationalen Finanzkapitals, die an und für sich selbstverständlich die Massen der Arbeiterschaft und der werktätigen Bevölkerung in Stadt und Land niemals an den Nationalsozialismus binden könnte, wird deshalb in der äußeren Agitation und Propaganda der Hitler-Partei durch eine Demagogie von solcher Skrupellosigkeit und solchen Zynismus vers chleiert, wie sie kaum irgendeine andere bürgerliche Partei, nicht einmal der Sozialfaschismus, aufzubringen vermochte. So allein ist es erklärlich, daß eine Partei, an deren Spitze neben politischen Abenteurern, bankrotten Generalen und Hohenzollern-Prinzen, Fabrikanten, abgetakelte Pfaffen, kurz ein Gemisch reaktionärer Elemente steht, trotzdem vorübergehend Zuwachs gerade aus jenen Massen erhält, die in den ersten Stadien der Auflehnung gegen das kapitalistische System und gegen die bürgerliche Ordnung begriffen sind.

Die Entwicklung des Klassenkampfes in Deutschland - und danit die Entscheidung über die Frage, ob es den Kapitalisten gelingt, aus der wachsenden Krise noch einmal durch maßloses Elend und Hungersnot der arbeitenden Klassen das kapitalistische System herauszuretten, oder ob die Krise durch das Proletariat unter Führung der Kommunistischen Partei auf proletarischem Wege gelöst wird durch den Sturz des Kapitalismus -, diese geschichtliche Entscheidung hängt zu einem großen Teil davon ab, wie weit es der Kommunistischen Partei Deutschlands gelingt, den Nationalsozialismus zu schlagen. Während es vor einigen Monaten in diesem Kampf der KPD gegen den Faschismus einen gewissen Tempoverlust gab, der der Hitler-Partei sehr zustatten kam, haben das Zentralkomitee und das Polbüro der Partei in den letzten Monaten eine entscheidende Wendung der Partei herbeigeführt.

Es ist nicht zuletzt ein Erfolg dieses Kampfes der KPD gegen den Faschismus, wenn in den letzten Wochen die Verwirrung um die inneren Widersprüche im 
nationalsozialistischen Lager immer mehr anwachsen (Verschärfung des Gegensatzes zwischen der abgespaltenen Straßer-Gruppe und dem Hitler-Lager, Meuterei der Berliner "Standarten-Abteilungen" gegen ihren Führer Goebbels, zunehmende Gegensätze zwischen den proletarischen Elementen der faschistischen Organisationen und den Führern usw. usw.). ....

Der antifaschistische Kampf der deutschen Kommunisten aber ist zugleich das Kettenglied für die Herstellung der proletarischen Einheitsfront mit den sozialdemokratischen Arbeitern für die proletarische Revolution, die mit dem kapitalistischen System auch dessen faschistische Kolonnen zerschmettern wird.

[Internationale Pressekorrespondenz Nr. 77 vom 12. September 1930, S. 1915f.]

16.9. 1930

10.

Das Wahlergebnis für das gesamte Reich

..... Die „Rote Fahne“ schreibt:

..... Es bleibt die Frage zu beantworten, ob die Wählermassen der bürgerlichen Parteien durch den Übergang zu den Nationalsozialisten ihre Zufriedenheit mit dem bestehenden kapitalistischen System, ihr Einverständnis mit der Herrschaft des Finanzkapitals zum Ausdruck bringen oder nicht. Es ist vollständig klar, daß genau das Gegenteil der Fall ist. Millionen Werktätiger stimmen für die Nazis, um ihre Erbitterung über die kapitalistische Mißwirtschaft, ihren Protest gegen die Young-Sklaverei zum Ausdruck zu bringen.

Die Nationalsozialisten sind die gekauften Agenten des Finanzkapitals, die bezahlten Henkersknechte des Young-Plans. Ihre ganze Politik dient der Verteidigung des Kapitalismus gegen die drohende proletarische Revolution, der Durchführung des Young-Planes auf Kosten der werktätigen Massen. Die Faschisten, die mit 107 Mandaten in den neuen Reichstag einziehen, müssen unvermeidlich das Vertrauen ihrer 6,4 Millionen Wähler zerstören, ihre Erwartungen enttäuschen, ihre Forderungen mit Füßen treten. Darum trägt der Wahlerfolg Hitlers mit unentrinnbarer Sicherheit den Keim seiner künftigen Niederlage in sich. Der 14. September war der Höhepunkt der nationalsozialistischen Bewegung in Deutschland. Was nachher kommt, kann nur Niedergang und Abstieg sein.

Der Faschismus ist die letzte Karte der deutschen Bourgeoisie, die sie gegen die drohende Revolution der Arbeiterschaft ausspielt. Diese Karte ist aber zugleich die unsicherste. Der Faschismus, dessen ganze Mission die gewaltsame Verhinderung des proletarischen Umsturzes, die bewaffnete Verteidigung der Kapitalhelfer ist, bildet im heutigen Deutschland das lebendige Symptom für die Auflösung der bürgerlichen Gesellschaftsordnung. Das ist dex objektive geschichtliche Widerspruch, der den kommenden Bankrott des deutschen Faschismus besiegelt. . .

Wir sehen die ganze Größe der faschistischen Gefahr. Wir sehen aber auch die emporsteigenden antifaschistischen Kräfte des Proletariats, deren mächtigste unsere eigene Partei ist. Der deutsche Faschismus, der blutdürstig seine Hand nach der Staatsmacht ausstreckt, hat - so wie es bei allen historischen Wendepunkten das Schicksal der deutschen Bourgeoisie war - das Unglück, daß er ein paar Jahre zu spät kommt. In anderen Ländern entstanden die faschistischen Diktaturen nach der 
Niederlage der Revolution, in der „Frühjahrszeit" der kapitalistischen Stabilisierung. Die deutschen Nationalsozialisten schreiten in einer Situation zur Macht, wo die Weltreaktion unter den Stößen der Wirtschaftskrise wankt und die kapitalistische Stabilisierung zusammenkracht.

[Internationale Pressekorrespondenz Nr. 78 vom 16. September 1930, S. 1940]

11.

Der Sieg des Faschismus

.... Bis jetzt stellte trotz der Unterstützung bedeutender Kreise des Großbürgertums und der Großgrundbesitzer der Faschismus eine ziemlich vergängliche Kraft dar. Seit dem Ende des Vorjahres begann das Anwachsen der faschistischen Parteiorganisationen als Massenorganisationen, und es setzten ihre Wahlsiege ein. Dies bedeutete, daß dank der Krise ein beträchtlicher Teil des Kleinbürgertums, teilweise aber auch erwerbslose Arbeiterjugend ohne politische Vergangenheit auf die kapitalistischen Drahtzieher, die Hitler am Gängelband führen, hereinfällt. Aber ein derartiges Ergebnis, wie es die Wahlen erbracht haben, hatte niemand erwartet. Die Faschisten sind zur zweitstärksten politischen Partei in Deutschland geworden. Die Anzahl der für die Faschisten abgegebenen Stimmen ist von 800000 auf mehr als 6 Millionen Stimmen gewachsen. Die Geschichte des politischen Kampfes kennt nichts dergleichen, besonders in einem Lande mit einer alten politischen Diffenzierung, in dem es jeder neuen Partei sehr schwer fällt, sich an dem Tische, der von den alten Parteien besetzt ist, einen Platz zu erobern. Nichts ist bezeichnender als die Tatsache, daß über diese Partei, die im politischen Leben Deutschlands den zweiten Platz besetzt hat, weder in der bürgerlichen noch in der sozialistischen Literatur etwas gesagt wurde. Es ist dies eine Partei ohne Geschichte, die plötzlich im politischen Leben Deutschlands erstanden ist, wie sich dank dem Wirken vulkanischer Kräfte plötzlich eine Insel aus dem Meere erhebt.

Der Sieg der Faschisten verdient größte Aufmerksamkeit. Er wird in der Zukunft einer allseitigsten Analyse unterzogen werden müssen, aber schon heute kann man sagen, daß dieser Sieg den Keim größter Überraschungen für die Bourgeoisie im Schoße trägt. Die faschistische Partei wurde als eine neue Stütze des kapitalistischen Regimes geschaffen, als eine neue Stütze des Trustkapitals, das das Nahen großer Stürme fühlt. Aber die Massen, die ihr folgten, kamen nicht zur Unterstützung des Trustkapitals, denn dann hätten sie nicht der Deutschnationalen Partei, der Deutschen Volkspartei und der Staatspartei davonlaufen brauchen. Sie folgten der faschistischen Partei, denn sie verspricht ihnen die Rettung vom Weltkapital, das Deutschland durch den Young-Vertrag aussaugt, und von den Trusts Deutschlands. Es sind dies vor allem die kleinbürgerlichen Massen der Stadt und des Dorfes. Daß das Dorf keine geringere Rolle spielte als die Stadt, zeigen die Wahlergebnisse in Ostpreußen, wo die Faschisten die Deutschnationale Partei schlugen, obgleich sie mit ihr einen Block bildeten. Der Bauer hat sich gegen den Großgrundbesitzer erhoben und ist in großen Massen zur neuen Partei gekommen, die ihm irgendein neues „drittes Reich der Arbeit" vorgaukelt. Nichts ist bemerkenswerter als die Tatsache, daß das Trustkapital seinen neuen Lakai, die faschistische Partei, mit der Maske des Sozialismus ausstatten 
mußte. Es ist nicht verwunderlich, daß diese Maskierung auch einen Teil der jungen Arbeiter anlockte, die vor kurzem aus dem Dorfe gekommen sind, und ihrer gibt es in Deutschland jetzt Millionen, wie dies die Analyse der sozialen Zusammensetzung der Arbeiter derartiger Riesenwerke, wie es das Leuna-Werk und die Anilinfabrik in Ludwigshafen sind, beweist. . . . .

[Internationale Pressekorrespondenz Nr. 81 vom 26. September 1930, S. 1998]

3. 6.1932

12.

Die Nationalsozialistische Deutsche Arbeiterpartei

Von Hans Jäger (Berlin)

\section{Die soziale Zusammensetzung}

\section{Wandlungen der sozialen Struktur - Die Bourgeoisie.}

... Die soziale Zusammensetzung der NSDAP hat auch ihre Wandlungen durchgemacht. In der ersten Zeit überwogen die Mittelschichten bei weitem. Was bis 1923, als die Bewegung vorwiegend auf Bayern beschränkt war, das Hakenkreuz trug, das waren wildgewordene Spießbürger. Ein Blick auf die Unterführer der damaligen Zeit zeigt das. Streicher in Nürnberg kann auch heute noch nichts anderes, als in seiner "Sturmfahne" Tag für Tag in der hysterischsten Weise auf die Juden zu schimpfen. Im „Mutterland“ des Nationalsozialismus zog der Appell an die Arbeiter weniger. Daher hatte man auch dort in der SA keine unzuverlässigen rebellischen Elemente, daher stand der Gau Bayern treu zu Hitler, der wohl wußte, warum er die Leitung bis auf den heutigen Tag in München beließ und die Parteitage mit Vorliebe in Nürnberg abhielt. Daher auch stand man sich mit den bayerischen Industriellen von vornherein gut, über deren finanzielle Zuwendungen schon in allerfrühester Zeit bereits gesprochen wurde. Aber die Bourgeoisie bekannte sich damals noch nicht offen zum Nationalsozialismus, sie spendete ja nach allen Seiten, um sich alle Türen offenzuhalten. In der alten Partei überwogen die städtischen Kleinbürger und die Bauern, soweit sie nicht zur Bayerischen Volkspartei oder zum Bayerischen Bauernbund hielten, vor allem im Chiem-Gau, der Hitlers Heimat am nächsten ist und auch jetzt, im Gegensatz zum sonstigen Nachlassen der Hitlerbewegung in Bayern, durch seine hohen Stimmziffern für Hitler auffiel.

Eine grundlegende Änderung trat ein, als die Bewegung nach dem Norden verpflanzt wurde, also nach 1923. Hier traten die beiden Leitsätze der Bewegung, die "nationale Bewegung kann nur sein, wenn sie sozialistisch ist" und „die sozialistische Bewegung kann nur sein, wenn sie national ist", durch die Hitler sowohl Unzufriedene von rechts wie von links zu sammeln suchte und - den Klassengegensatz in die eigenen Reihen verpflanzte, von vornherein viel stärker in Erscheinung. Es ist fast überflüssig, zu betonen, daß das "Sozialistisch“ ebenso wenig mit Sozialismus wie das „National“ mit wirklicher nationaler Befreiung zu tun hat. Im Norden setzte man sich im Kampf gegen die deutschvölkische, bis 1923 mit Hitler lose verbundene Bewegung durch, die zwar auch nationalistisch-faschistisch-antisemitisch, aber offen reaktionär, monarchistisch, offen arbeiterfeindlich war, nur die Großgrundbesitzer und adligen Junker, die Mächte von gestern, vertrat, also einer sozialen Demagogie 
keinen Spielraum ließ. Diese Deutschvölkischen wurden von den Nazis so gut wie zertrümmert. Es muß einer späteren Untersuchung vorbehalten bleiben, zu erklären, wieso diese Bewegung später in diesen Gebieten unendlich mehr Fortschritte machen konnte, während ihr Aufschwung an den eigentlichen Ausgangspunkten ein unvergleichlich langsamerer ist, ja wieso diese aus dem katholischen Süden stammende, großenteils von Katholiken (Hitler, Goebbels, Gregor Strasser, Epp) geführte Bewegung ausgerechnet in den katholischen Gebieten an Intensität nachläßt. Wenn sie auch dort natürlich stark ist und in die Reihen des Zentrums (Westfalen, Rheinland, Schlesien) stark eingebrochen ist, so steht das immerhin in keinem Vergleich zu den Nordgebieten, wo Hitler z.B. Hindenburg bei der Reichspräsidentenwahl schlug (Pommern, Holstein). Ohne etwa dies überschätzen zu wollen, darf man doch die religiös-ideologische Bedeutung für die Auseinandersetzungen in Deutschland nicht gering einschätzen. Das zeigte sich ebenso bei der Trennung des Generals Ludendorff und des Rassefanatikers Dinter von Hitler (Begründung: er habe seinen Frieden mit Rom gemacht und den Kampf gegen die schwarze Internationale eingestellt), als auch bei dem Widerwillen, auf den eine Koalition der Nazis mit dem Zentrum in der Mitgliedschaft stößt.

Die scheinsozialistische Demagogie, die im Norden also viel stärker betrieben wurde, hatte nun verschiedene Folgen: Zunächst einmal gewann man hier in ganz anderem Umfange rückständige, vor allem durch Arbeitslosigkeit moralisch zermürbte, deklassierte Arbeiter, wie wir noch sehen werden, als in Bayern, und speziell die SA, wies eine andere Zusammensetzung auf. Dann aber waren große Teile der Bourgeoisie im Anfang erklärlicherweise mißtrauisch und dadurch weniger gebefreudig als in Bayern. Sie nahmen entweder die sozialistischen Phrasen selbst für bare Münze oder fürchteten, daß sie von den Anhängern für bare Münze genommen werden könnten. Es war die Zeit, wo Hitler noch nicht seine Reden vor den Wirtschaftsführern gehalten und sie über seine wirtschaftlichen Pläne beruhigt hatte, wo er noch nicht so fest im Sattel zu sitzen glaubte, daß er sich solche Selbstentlarvung ungefährdet gestatten konnte. Der Vergleich zu Italien liegt auf der Hand, wo Industrielle auch eine Zeitlang mit Mißtrauen die faschistische Bewegung, ihr Gerede von Klassenkampf und RevoIution, ihre Zustimmung zur Besetzung der Fabriken verfolgten, die Taschen zuhielten und die neue Partei für eine unzuverlässige hielten. Um diese Zeit finden wir nur kleine Unternehmer bei Hitler, die auf dieser Plattform ihre Sorte von Klassenkampf gegen die großen Trusts, das Finanzkapital, die Börse durchführen zu können glaubten (Fertigindustrielle gegen Rohstoffmonopolisten).

Die geschilderte Form der Propaganda : „die nationale Bewegung muß sozialistisch, die sozialistische Bewegung muß national werden!", in Norddeutschland, besonders von Otto Strasser und Goebbels, später auch von Dr. Ley betrieben, hatte aber noch weitere Folgen: In der Mitgliedschaft bildeten sich - ebenso wie in der SA - große Gruppen, die an den Sozialismus so sehr glaubten, daß sie später rebellierten (teilweise wurden sie von Otto Strasser und Stennes aufgefangen, teilweise gingen sie bereits zur KPD), oder aber fest davon überzeugt waren, daß Hitler den Sozialismus bringen werde (das sind also die Rebellen von übermorgen, die noch zum Führer Vertrauen haben) und alle seine Handlungen nur einer bestimmten Taktik zuschreiben, die man aus Disziplin nicht durchkreuzen dürfe. . . .

Unter der Führerschaft bildeten sich dann, abseits der Hitler-Clique, Gruppen, die den Bruch mit Hitler vollzogen (Otto Strasser und Stennes), um die Anhängerschaft 
aufzufangen und an dem Überlaufen zur KPD zu hindern, darüber hinaus Tuchfühlung über Ehrhardt und den Stahlhelmer Treviranus mit Brüning suchten. Ein Teil der Stennes-Gruppe sagte sich im letzten Augenblick von ihr los und blieb Hitler „treu“ bzw. ließ sich von ihm kaufen (Goebbels ließ für den Posten des Reichspropagandaleiters und Gauführers von Berlin die "Linken“ im Stich), die aber nun innerhalb dex Partei weiter opponieren, und zwar um die Mitglieder vor einem Austritt zurückzuhalten, um sich, gestützt auf den "sozialistischen " und protestantischen Norden, durch eine Scheinopposition (gegen die Harzburger Zusammenarbeit mit den Deutschnationalen, gegen eine Zentrums-Koalition) gegenüber dem legal gewordenen, parlamentsfrommen, koalitionshungrigen, außen- und innenpolitisch verständigungsbereiten, putschfeindlichen, reparationsfreudigen, gar zu deutlich mit der Industrie liebäugelnden, ständig zaudernden „Führer“ eine Position zu verschaffen.

Das ist ein Spiel mit verteilten Rollen, nichts als ein taktischer Gegensatz: Hitler tut alles, um die Bourgeoisie nicht vor den Kopf zu stoßen (er verbot Goebbels die demagogische, für Berlin geplante Feier am 1. Mai, „deutsche Maifeier", er war entsetzt über die Vorfälle auf der Leipziger Straße im Oktober 1930, als in den Waren- und Kaufhäusern die Auslagen eingeschlagen wurden, er entschuldigte sich wegen der Beteiligung am Metallarbeiterstreik, der ja auch faktisch sabotiert wurde), aber auch Goebbels tut alles, um mit Hilfe einer schamlosen Demagogie Arbeiter zu behalten und zu gewinnen. Während Hitler mit den Parteien verhandelt, erklärt sein Gegenspieler, sie gehörten sämtlich auf den „Misthaufen der Geschichte“, während Hitler mit dem Zentrum liebäugelt, greift es Goebbels aufs heftigste an, während Hitler mit den Deutschnationalen flirtet, erklärt Goebbels, man werde es wie in Italien machen, wo Mussolini die Nationalpartei später auch an die Wand drückte und verschluckte (dabei hat übrigens diese schwerindustrielle Federzoni-Gruppe später dem ganzen Faschismus den Stempel aufgedrückt und den wildgewordenen Kleinbürgern der Farinacci-Gruppe jede Politik auf eigene Faust untersagt).

So sieht die soziale Zusammensetzung schon ab 1926 anders aus als zuvor, und im Norden anders als im Süden.

Eine weitere Etappe war dann, daß man auch im Norden die Großunternehmer von der Ungefährlichkeit, ja von der ungeheuren Nützlichkeit dieser nun über das ganze Reich ausgedehnten Partei überzeugte, die schon 1928 bei den Wahlen alles aufsaugen konnte, was vorher die Völkischen innehatten. Planmäßig wurde, gewissermaßen mit Augenzwinkern, der Bourgeoisie klargemacht, daß sie gefälligst die Worte "Sozialismus" und „Arbeiterpartei " in Kauf nehmen und nicht ernst nehmen müßten, daß sie auch Goebbels gewähren lassen sollen, daß sie ein Interesse an dieser Art Propaganda hätten, durch die allein die Arbeiter dem Marxismus entrissen würden. Und als sich die SA zu einer Terrortruppe nicht gegen jüdische Kapitalisten, sondern ausschließlich gegen die revolutionäre Arbeiterschaft, gegen die verhaßten Kommunisten entwickelte (gegen die man die unklar sozialistisch denkenden SA-Leute unter der Vorspiegelung "Mörder eurer Kameraden“ und "Knechte des Finanzkapitals“ sowie unter der Losung "Tod dem Marxismus" aufhetzte), da waren die Unternehmer beruhigt. Erst recht, als sie sahen, daß sich die „weißen Zellen“, die NSBO (Betriebsorganisation der Nazis) als Denunzianten der Roten, als Streikbrecher, als Mittel zur Niederhaltung der Belegschaften verwenden ließen. Und vollends erst recht, als die Bourgeoisie sich bewußt wurde, hier liege eine unerhörte Kraftreserve vor, die man in dem Maße, wie die SPD, kompromittiert durch ihre Taten, "abgenutzt" werde, 
innenpolitisch zur Errichtung der Diktatur im Zeichen der zunehmenden Krise verwenden und gleichzeitig außenpolitisch wenigstens als Drohung, als Trumpf bei Reparationsverhandlungen benutzen könne. . . .

Die Unternehmer sind nicht nur Geldgeber (Borsig), sondern auch Mitglieder geworden, wenn man auch in verschiedenen Fällen die Aufnahme sorgsam verschwieg, ja sogar dementierte (Thyssen und der ehemalige Reichsbankpräsident Schacht). Es gibt eben auch Mitgliedskarten, die im Braunen Haus deponiert sind und von denen die anderen nichts zu wissen brauchen. Von sonstigen „Freunden der Arbeiterpartei“ nennen wir aus den Reihen der Ruhrindustriellen Kirdorf, Wiskott, von der Textilindustrie Mutschmann, über dessen Einfluß auf Hitler der ausgetretene Kapitänleutnant Mücke große Enthüllungen machte. Andere Industrielle beschränken sich, von den Deutschnationalen, der Volkspartei oder den Volkskonservativen aus den Nazis gegenüber eine mehr als wohlwollende Haltung einzunehmen. Die Blätter der Schwerindustrie, wie „Deutsche Allgemeine Zeitung“, „Börsenzeitung“, „RheinischWestfälische“, „Bergisch-Märkische“, „Bergwerkszeitung" usw. sind ja bereits so nazifreundlich, daß sie sich nur durch die dem "Angriff“ (Organ Goebbels) überlassene soziale Demagogie von der Nazipresse unterscheiden. Man legt natürlich Wert darauf, diese Unternehmer nicht allzusehr herauszustellen, um den Kurs auf die Arbeiter nicht zu gefährden. Spielen sie aber, wie Mutschmann, in der Partei eine Rolle, dann sind es eben die "schaffenden", die "arischen" Unternehmer, die man dadurch schmackhaft macht, daß man auf ihre „soziale Gesinnung" hinweist. (Schon dies wird als "Sozialismus" deklariert und eine geheuchelte Kameradschaftlichkeit, die Gnade, eine Zigarette oder Freibier abzubekommen oder mal mit im Auto fahren zu dürfen, wird von ungeschulten Elementen als Zeichen „sozialistischen Denkens und wahrer Volksgemeinschaft" angesehen.) Dahinter verbergen sich dann oft nur dürftig Kasernenhofton und Herrenmenschentum. Der eingepaukte Kadavergehorsam und der militärische Drill, die fehlende Schulung, die eigene Notlage und die mangelnden politischen Erfahrungen gerade der Jüngeren verhindern dann oft, hinter die Maske zu sehen.

Viel ungenierter hat man auch die Großgrundbesitzer herangeholt, wie von Wangenheim, Corswandt in Pommern. Hier glaubte man, sich den Bauern gegenüber gar keine Reserve auferlegen zu müssen. Die neue preußische Landtagsfraktion der Nazis weist eine Unmenge von Adligen auf, u. a. übrigens auch aus dem katholischen rheinischen, westfälischen und schlesischen Adel (wie von Eltz-Rübenach), dem das Zentrum noch nicht faschistisch genug erschien, weil es doch nach 1918 Linkstendenzen heucheln mußte. Zu ihren Prunkstücken gehört der Hohenzollernprinz Auwi.

In der NSDAP gibt es dann eine reiche Auslese an all denen, die mittelbax zur Bourgeoisie gehören. Es wimmelt hier von hohen Beamten, von Industriedirektoren und Unternehmersyndici, die wohl erkannt haben, daß die NSDAP die „hohe Aufgabe" hat, den Arbeitern wieder Respekt und Autorität beizubringen, von hohen Generalen (Litzmann, Epp). Und ihnen reihen sich die Konjunkturritter an, die den Anschluß nicht verpassen wollen und sich einen Platz im Dritten Reich sichern, ihre Positionen nicht gefährden wollen.

\section{Mittelschichten und Proletariat}

Aus den Reihen der Bourgeoisie hat die Partei aber noch keine Massen. Diese liefern in erster Linie die Mittelschichten. Trotz des proletarischen Anhangs, den man 
anfangs unterschätzte, weil man fälschlich von dem bayerischen Ausgangspunkt aus verallgemeinerte, bleiben sie das Kernstück. Ganz genau wie in Italien. Trotz des unleugbaren Einbruchs in die Arbeiterschaft und vor allem in die Reihen der Erwerbslosen (besonders Jugendliche), der in manchen Bezirken nicht unerheblich ist (HalleMerseburg und Chemnitz-Zwickau, neuerdings aber auch Berlin und Hamburg, Ruhrgebiet und Oberschlesien), trotz des geradezu dominierenden Einflusses auch auf zwei wichtige Gehaltsempfängerkategorien, nämlich die Angestellten und die unteren Beamten, bleibt der sogenannte Mittelstand das Kernstück. Es wäre eine maßlose Übertreibung, wenn man das verkennen und jetzt in der Bewertung des proletarischen Sektors von einem Extrem ins andere fallen wollte.

Die Mittelschichten gaben den geeigneten Boden ab. Sie sind ja die „Brücke“, die der Nationalsozialismus zwischen Unternehmern und Arbeitern braucht, zur „Herstellung der Volksgemeinschaft". Proletarisch in der Lebensführung, bürgerlich im Denken, sich sträubend gegen das Versinken im Proletariat und immer noch träumend von der Zugehörigkeit zur Bourgeoisie, mit der sie sich früher etwa durch den gemeinsamen Besuch der höheren Schule, die „Erlaubnis", Reserveoffizier werden zu dürfen, den Besitz von 1-2 Aktien verbunden fühlten und der sie es, mag auch der Magen schreien, wenigstens in Kleidung und - Sommerreise gleich zu tun suchen. Ihre Traditionen (christlich und national), ihr Schuldrill (Verständnislosigkeit gegen wirtschaftliche Dinge, Überbetonung des Außenpolitischen, Anbetung der großen Führerpersönlichkeit, Geringschätzung der Unterklasse, die man mit Lumpenproletariat gleichsetzt, gerade in Deutschland), ihre politische Ungeschultheit (früher hatte doch der Kaiser für alle gedacht), all das machte sie geradezu reif für den Nationalsozialismus in einer Zeit der fürchterlichsten Krise von ganz anderen Ausmaßen als zur Zeit der Industriealisierung Deutschlands, wo zwar die Handwerker der industriellen Konkurrenz zum Opfer fielen, die Kleinhändler teilweise durch die Warenhäuser Schaden litten, bei dem Aufstieg des deutschen Imperialismus aber noch genug Existenzmöglichkeiten vorhanden waren. Jetzt wütet die Konzentration des Kapitals ganz anders in Trust-Deutschland, gehen die Konkurse in die Zehntausende, brechen die Kleinbetriebe durch Steuern und eingeschrumpfte Kaufkraft reihenweise zusammen, müssen Handwerker und Kleinhändler, ohne wie etwa noch 1923 in den Fabriken Aufnahme finden zu können, stempeln gehen, Wohlfahrtsunterstützung beziehen oder den Gashahn öffnen. Nicht anders ergeht es den freien Berufen. Künste sind Luxus geworden. Maler, Bildhauer, Journalisten und Schriftsteller haben in der deutschen Republik keinen Platz mehr. Viele unter ihnen, Schriftsteller wie Hans Heinz Ewers, Bronnen, Johst, Jungnickeil, Schauspieler wie Gebühr und Werner Krauss wittern die Konjunktur. Aber die anderen packt das Grauen. Ebenso die deutschen Intellektuellen, der Nachwuchs, die Studenten, die in Jahrzehnten keinen Arbeitsplatz finden können, die Junglehrer, die zu Tausenden abgebaut wurden, die Angestellten, die in Büro und Bank Opfer der Rationalisierung wurden und zu Hunderttausenden arbeitslos wurden oder nur noch Hungerlöhne beziehen, nicht minder die unteren Beamten, denen es genauso ergeht.

Sie stehen ratlos. Die kommunistische Propaganda konnte sie bis jetzt nur teilweise erfassen. Die meisten von ihnen werden zum erstenmal in das politische Leben einbezogen. Die Kommunisten verstehen es noch nicht, an sie richtig heranzukommen. Der Nationalsozialismus zwingt sie weniger, nachzudenken als die kommunistische Agitation es tut. Hitler verheißt die Rettung! Es muß anders werden. Jawohl! Er 
verheißt allen Brot, Aufhören der Krise, Sinken der Steuern! Der starke Mann wird helfen, er bringt etwas ganz Neues, er ist der Erlöser, der Messias. Sein Reich ist aber von dieser Welt. Liegt in der Vergangenheit. Es ist das schattenlose Bild des BismarckReiches, des Kaiserreiches, in dem es Geschäft und Extraprofite, Ämter und Würden und für den deutschen Arbeiter Arbeit gab. Dieses Reich soll auferstehen als Drittes Reich. National, das findet bei diesen Schichten Widerhall, im Gegensatz zu den Erfüllungspolitikern, zu Demokraten und Sozialdemokraten, die man fälschlich als Pazifisten und Internationale ansieht. Frankreich ist schuld! Die Reparationen, Versailles, der Young-Plan! Was denn sonst? Doch nicht etwa das kapitalistische System! Die Wirtschaftsordnung! Die hat doch früher so gut funktioniert, ehe das Zentrum und die "Marxisten" kamen, unter der Monarchie, ehe die Republik kam. System können sie sich nur mit Personen verknüpft vorstellen, also „System Brüning“, "System Braun“. Was heißt kapitalistisches System? Etwas anderes gibt es doch nicht. Rußland? Nein, das bedeutet Mord, Kirchenverfolgung, Hunger, "Teilen“ dessen, was man hat, Gleichmacherei. Nur das nicht! Also ein Personenwechsel! Das Wirtschaftssystem soll bleiben! Unter dem kann man bei besserer Regie doch mal vielleicht selbst wieder Kapitalist spielen, wie man es früher tat, oder wieder so leben wenigstens wie früher. Frankreich ist also schuld! Und die Marxisten sind schuld, die den Dolchstoß führten, uns an Frankreich auslieferten. Und die Juden! Denn sie sitzen bei den Marxisten, den Vaterlandsverrätern, sie sind die Nutznießer des heutigen Staates, als Regierungsleute ebenso wie als Warenhausbesitzer, die den Mittelstand kaputt machen, als Schuldige an der Inflation, die alle enteignete, als Börsenschieber, als Bankiers. Juden, die durch die Marxisten hereingelassen wurden wie Barmat und Sklarek, die mit den Marxisten zusammen Geschäfte machten und das Volk ausplünderten. Das ist der einfache Gedankengang. Aber Hitler wird die Marxisten verjagen, die das Land den Juden und den Franzosen auslieferten, dem internationalen Finanzkapital versklavten.

Bei den Angestellten wurde diese Agitation noch dadurch erleichtert, daß diese auch infolge mangelhafter politischer Schulung großenteils nicht willens waren, sich in die Klassenfront des Proletariats einzureihen, und ihr Antisemitismus war nichts weiter als ein verballhornter, irregeleiteter Klassenkampf, denn sie stehen in Handel und Bank mehr jüdischen Arbeitgebern gegenüber, als die Arbeiter in der Industrie. Ähnliches gilt von den Mittelschichten auf dem Lande, von den Bauern, unter denen der Nationalsozialismus ebenso ungeheure Erfolge zu erzielen vermochte wie unter den Mittelständlern, den Angestellten, den Intellektuellen. Das gilt ebensowohl von den Großbauern, die sich vom Nationalsozialismus dasselbe versprachen, wie die städtischen Unternehmer, als auch von den Kleinbauern. Sie wurden von Steuern erdrückt, die geschwundene Kaufkraft wirkte sich auch für sie aus. Tausende von Zwangsvollstreckungen kennzeichneten den Ruin der deutschen Landwirtschaft. Darunter hatten ehemals reiche Bauern wie die Viehzüchter von Holstein und Nordhannover genauso zu leiden wie die von jeher notleidenden armen Bauern der Eifel oder der Rhön. Der Bauer war für alle das Lasttier. Ihm wurden alle Lasten aufgebürdet, weil er sich nicht zu wehren vermochte, selbst zu einer Zeit, als die Bourgeoisie sich gegenüber der Arbeiterklasse noch in der Defensive befand. Auf ihn nahm die Sozialdemokratie ebensowenig Rücksicht wie auf den Handwerker. Mochte er alles tragen, wenn nur die Bourgeoisie geschont wurde. Da kam Hitler. Seine Beweisführung, die augenblicklichen Regierenden seien an allem schuld, war primitiv, aber 
einleuchtend. Marxisten, Juden, Franzosen! Dem Antisemitismus war der Bauer durch seine Erlebnisse mit dem Getreide- oder Viehhändlex sowieso leicht zugänglich. So gewann der Nationalsozialismus ebenso den Bauern des Ostens, dem er vorgaukelte, die Regierung wolle ihn nicht gegen Polen und Litauen schützen, und sei allein schuld an seiner Not, den Bauern des Nordens, der sich durch Terrorakte (Sturm auf Finanzämter, Kampf gegen Versteigerungen, Boykottbewegung) zu wehren suchte (Hannover, Holstein), den Winzer an der Mosel, den die Trustherren beim deutschspanischen Handelsvertrag um industrieller Interessen willen preisgegeben hatten, den Weinbauern der Pfalz, dem man von der Franzosenzeit sprach und den man nationalistisch aufpeitschte. Die Terroristen hatte Hitler zwar um seiner Legalität willen preisgegeben, wie Claus Heim, aber das dadurch entstandene Mißtrauen, das sich in der Abwanderung zu Otto Strasser und Ludendorffs Tannenbergbund äußerte, hat Hitler schon wieder überwunden. Die gesteigerte kommunistische Landpropaganda suchte der Nationalsozialismus durch einen wachsenden Terror (bekannt ist die Schlacht bei Albersdorf in Holstein, wo sich die Arbeiter gegen verhetzte Bauern in stundenlangem Kampf wehren mußten) und durch eine unerhörte Lügenhetze zu vereiteln. Dabei spielten die absichtlich falsch dargestellten und verallgemeinerten Maßnahmen gegen die Kulaken in der Sowjetunion, seine verzerrte Berichterstattung über die Kollektivisierung, Greuelmärchen, die Frage der Gottlosenbewegung, die falsche Darstellung der Lage der Wolgadeutschen eine Hauptrolle, und wo alles nichts half, da bediente man sich der plumpesten Fälschung, indem man von angezündeten Bauernhöfen sprach. Die jungen Bauernsöhne, Landarbeiter, Gutsknechte, Tagelöhner wurden in die SA geholt. Nötigenfalls setzte man sie auch in den Städten ein bei größeren Aktionen oder in solchen Fällen, wo man den proletarischen SA-Mitgliedern nicht genügend traute. Kamen die Landarbeiter nicht gutwillig, so halfen die Besitzer und Inspektoren nach, und machte ein Bauer nicht mit, so wurde der Boykott über ihn verhängt.

Die Nazis haben jedoch auch große Erfolge erzielt unter den Berufsbeamten, sowohl unter den oberen wie auch unter den unteren. Planmäßig wandte man sich zuerst an die Finanzbeamten, dann an die Beamten der Post und Eisenbahn, dann aber auch an sämtliche Beamte ob sie nun bei den Gemeinden, in Ländern oder im Reiche Dienst hatten - in den Gerichtskanzleien oder bei der Polizei. Der wiederholte Gehaltsabbau hat die unteren Beamten den Nazis geradezu zugetrieben, bei den oberen Beamten ist die Hetze gegen die „Parteibuchbeamten“ auf fruchtbaren Boden gefallen. Die Sozialdemokratie hat nach der Revolution von 1918 den ganzen monarchistischen Beamtenapparat intalst gelassen und ihm nur eine Anzahl der eigenen Anhänger zugeteilt, um diese zu versorgen. Diese „Parteibuchbeamten“ wurden zu einem starken Agitationskampf der Nazis, die versprachen, daß die Berufsbeamten von dieser lästigen Konkurrenz befreit werden und die alte Geltung wieder erlangen sollen. Hand in Hand damit arbeiteten die Nazis mit Drohungen, Einschüchterungen, schwarzen Listen. Als nun der Nationalsozialismus erstarkte und Aussicht bekam, die Macht zu erlangen, da stellte sich schon deswegen eine Unmasse von Beamten um, um den Anschluß nicht zu versäumen. Der Beamtenanhang hat aber für die Nazis auch eine große politische Bedeutung - sie haben dadurch wichtige Machtpositionen in Händen.

Was fesselte nun die Arbeitslosen an die NSDAP? Wollen sie etwa eine faschistische Diktatur? Sind sie auch so nationalistisch? Das zum Teil. Aber all das ist nicht das Entscheidende. Der Weg, um zu Hitler zu kommen, ist ein verschiedener. Den einen 
enttäuschte die SPD, den anderen das Zentrum, einen dritten gar die Deutschnationalen, wieder ein anderer war vorher ein unbeschriebenes Blatt. Der eine brachte in erster Linie das Vertrauen zu Hitler mit, der andere schiebt alles auf die Juden, der dritte auf den Young-Plan, der vierte auf die „Marxisten“. Der eine haßt die Kommunisten, der andere hat daran gar kein Interesse und glaubt nun bei Hitler an die schnellere Erreichung des Zieles, und gar unter den Nazi-Wählern denkt sogar mancher, dadurch nur das Chaos und den Zusammenbruch schneller herbeizuführen. Im Herzen sind sie aber alle irgendwie revolutionär, in einem anderen Sinne als die Kleinbürger, bzw. sind es geblieben. Sie sehen im Nationalsozialismus die Waffe gegen das Kapital, genau wie das Kapital in ihm die Waffe gegen das Proletariat sieht.

Man kann diesen grotesken Zustand nur verstehen, wenn man sich in das Maß von Verzweiflung, Hoffnungslosigkeit, Zermürbung und Ungeduld hineinversetzt, das die erfaßt, die teilweise nur noch 6 bis 8 Mark die Woche haben. Da erscheint Hitler für sie als der letzte Rettungsanker, man klammert sich an den Strohhalm. Diese Verzweiflungsstimmung hat seit einigen Monaten rapid zugenommen. Diese Verzweifelten laufen zu den stärkeren Bataillonen, deren gesteigerter Einfluß einen Erfolg in kürzester Frist verheißt und deren Opposition von ihnen ebenso wie alle Versprechungen geglaubt wird. Die Zahl blendet, die Suggestion tut ihre Wirkung. Aus 6 Millionen wurden 11 dann 13. Bald werden es vielleicht noch mehr sein. Die Erwerbslosen, die jetzt kommen, ob jünger oder älter, mit ihren Frauen, das sind keine Feinde der Kommunisten, sie sind noch nicht einmal so verhetzt wie die früheren, älteren Mitglieder, sie denken nur: Bei den Kommunisten sind nur 4 Millionen. Das dauert zu lang. Hier aber wurde die Hilfe für einen bestimmten Tag verheißen: 13. März bei der Präsidentenwahl, 24. April bei der Preußenwahl, 24. Mai bei der Landtagseröffnung. Jetzt vertagt man wieder auf Oktober!

Aber es sind nicht nur die Versprechungen. Man tut auch etwas! Denn man hat ja die Millionengelder, über deren Herkunft sich die Mitglieder keine Gedanken machen. Man gibt den arbeitslosen SA-Leuten, die vorher zerrissen herumliefen, Schuhe und Kleider. Man gibt ihnen zum Teil Suppen und Verpflegung, Zigaretten, Bier und Taschengeld. Ist das nicht - „Sozialismus“? Man kauft die jungen Erwerbslosen unmerklich. Sie werden sich dieses feinen Betrugs nicht bewußt, denn man macht es ja so, daß er als Lohn für treue Dienste aussieht. Sie denken, wenn sie jetzt schon solche Fürsorge ernten, wie erst nach der Machtergreifung? Sie wissen nicht, daß da nur die Peitsche übrig bleibt und daß man sie mit den Bettelsuppen und Gamaschen kaufte. Ein Teil freilich ist so deklassiert, daß er dadurch schon bewußt zum käuflichen Landsknecht wurde. Aber nicht alle. Wir müssen unterscheiden lernen.

Das alles wäre unvollständig, wenn man nicht auch von dem proletarischen Anhang spräche. Es wurde schon davor gewarnt, hier mit falschen Maßen zu messen. Er ist w'eder so klein, daß man ihn als belanglos vernachlässigen könnte, noch so groß, daß man von einem wesentlichen Einbruch in die Reihen der Arbeiterschaft sprechen könnte. Von 1928 an war ein stärkerer Einbruch zu verzeichnen, besonders in Sachsen und Thüringen (Erzgebirge, Vogtland), also in der Gegend der Klein- und Mittelbetriebe, der Heimarbeit und Hausindustrie, gleichzeitig aber auch in den Gegenden der größten Not, der furchtbarsten Verelendung, der Kulilöhne und der prozentual schärfsten Erwerbslosigkeit, und dieser Einbruch kam etwa 1930 zum Stehen. Von da ab sah man, wie die Nazis mehr von den bürgerlichen Parteien profitierten. Seit 1932, seit dem sprunghaften Zunehmen der Krise, ist aber ein neuer Einbruch leider 
unleugbar. Jede Vogel-Strauß-Politik ist da fehl am Platze. Schon die Tatsache, daß der Nationalsozialismus hauptsächlich auf Kosten der bürgerlichen Parteien zunahm, darf nicht mißdeutet werden. Denn dort gewann er doch auch - Proletariat, und zwar zuerst! Diese wanderten zuerst aus den Reihen der Deutschnationalen ebenso wie aus dem Stahlhelm ab, mit der Begründung, das seien Knechte des Trustkapitals, weil diese das Tarnen schlechter verstanden als die Nazis. Diesen prolctarischen Anteil leugnen, hieße doch, da die bürgerlichen Parteien die Mehrheit haben, leugnen, daß das Proletariat zahlenmäßig am stärksten in Deutschland ist. Von den Angestellten wurde schon gesprochen. Wir beschäftigen uns hier nur mit der Arbeiterschaft. Gewiß überwiegen hier die Jüngeren, über die wir noch gesondert sprechen werden. Der proletarische Nachwuchs stellt einen ganz besonders hohen Prozentsatz der Anhängerschaft der Nazis, und hier liegt eine ungeheure Gefahr. Natürlich ist das Eindringen in die Reihen der Arbeiterschaft auch lokal verschieden, hier mehr als dort, am bedenklichsten vielleicht in Chemnitz, Plauen, Bochum, Gelsenkirchen, aber auch feststellbar in Berlin, Hamburg, Essen, Köln, Breslau, Hannover. Und zwar gilt das zum Teil auch von den Betriebsarbeitern (Bergbau, Metallindustrie, vor allem aber Kleinbetriebe, Zwergbetriebe), ganz besonders aber von den Arbeitslosen.

Man hat mit Recht gesagt, daß man aus der Zusammensetzung der SA keine falschen Schlüsse ziehen dürfe. Gewiß, dort überwiegt der proletarische Bestand (im. Gegensatz zur SS), denn der Gemüsehändler, der Postsekretär, der Reisende, sie überlassen diese Arbeit lieber den Proleten. Aber auch in der Partei geht die Zahl in die vielen Tausende, unter den Wählern ist sie mit 2 bis 3 Millionen nicht zu hoch veranschlagt.

Wo kommen sie nun her? Sowohl von rechts, wo sie sich in den alten bürgerlichen Traditionsparteien nicht mehr wohlfühlen, aber die passende gelbe Ideologie, den Nationalismus und auch schon ein Körnchen Antisemitismus mitbrachten, schon reif für diese Sorte "Sozialismus" (obgleich dieser Schritt auch bei ihnen bereits eine wenn auch verworrene klassenmäßige Rebellion darstellte), teils aus den christlichen Gewerkschaften (Deutschnationaler Handlungsgehilfenverband, Landarbeiter usw.), teils aus den gelben Werkgemeinschaften, Arbeitervereinen, als auch von anderen Arbeiterkreisen. Hier zeigen sich die Auswirkungen der sozialdemokratischen Politik. Arbeiter wurden am Sozialismus, am Marxismus überhaupt, an der Internationale, an allem irre. Sie ließen sich zum Nationalismus bekehren oder nahmen ihn unwillig in Kauf. Ihr Übergang war nur eine logische Folge des Handelns von Noske und Hörsing. Winnig und die Altsozialisten hatten dies vorgemacht, denen heute die Konjunkturritter folgen. Diese Arbeiter verkannten, daß es noch eine andere Kraft gibt, die gegen diesen Staat ankämpft, die KPD. Und die Sozialdemokratie hat sie auf dem Gewissen. Es kamen aber auch Arbeiter, die links von der Sozialdemokratie standen. Kein Zufall, daß dies gerade dort der Fall war, wo die kommunistischen Oppositionsgruppen die Partei zu schädigen suchten (Sachsen, Thüringen). Dies schuf Verwirrung, Verärgerung, Ratlosigkeit. Brandler und die Trotzkisten wurden zum Zutreiber der Nazis, gegen die sie angeblich bessere Rezepte haben wollen. Das Verbot des RFB wirkte unter dem Teile der politisch ungeschulten Anhänger auch so, daß sie, dumpf antikapitalistisch, aktivistisch und auf Kampf eingestellt, glaubten, diesen Kampf nun dort fortsetzen zu können, wo ihnen hier die Möglichkeit genommen war. Als Schläger gegen ihre ehemaligen Parteigenossen taten sie sich in der SA besonders hervor, und bei allen Prozessen der letzten Zeit tauchen immer wieder diese ehemaligen 
Rotfrontkämpfer auf. Verzweiflung, Ungeduld, mangelnde Klarheit, Unglaube an die Revolution ließen sie auf den „Führer" hoffen, ließen sie an das „revolutionäre“ Ziel, den "Sozialismus" glauben und auf baldige Aktionen vertrauen. Es geht dort schneller! Das war die Devise. Das waren also nicht Gelbe, also willkommene Proleten, sondern höchst unerwünschte, deren Eintritt Hitler sehr ungern sah, vor denen ihm angst und bange wurde, die er durch die Stennes-Revolte bereits loszuwerden wünschte, die aber Goebbels in richtige Nazis umzuwandeln sich durchaus zutraute und die ihm als Aktivisten erwünscht waren. Sie sind der Unruheherd in den SA, sie sind der Bourgeoisie nicht lieb, sie sind ungebärdig, sie stecken die anderen an. Daß diese Leute bei den Nazis sind, ist eine Folge der mangelnden Schulung, der Ungeduld. Sie glauben, daß Hitler gegen das System kämpfe und machen dabei den entgegengesetzten Fehler wie die, die meinen, daß die Sozialdemokratie gegen den Faschismus kämpfe.

Das ist also die Mitgliedschaft: an der Spitze die Unterdrücker, die eine Bürgerkriegsarmee aus den Unterdrückten sammeln (solchen Unterdrückten, die sich dazu aus Unwissenheit hergeben, solchen, die sich kaufen ließen, und solchen, die sogar ehrlich glauben, hier gegen die Unterdrücker zu kämpfen), und im Gefolge die Verzweifelten aus Mittelschichten und Proletariat, aus Kleinbürgern, Bauern, Studenten und Arbeitern, aus Erwerbslosen aller Schichten, Notleidende und völlig Deklassierte (dazu gehören auch viele Mittelständler), Hungernde und Verhungernde.

[Internationale Pressekorrespondenz Nr. 46, vom 3. Juni 1932, S. 1427-1431] 